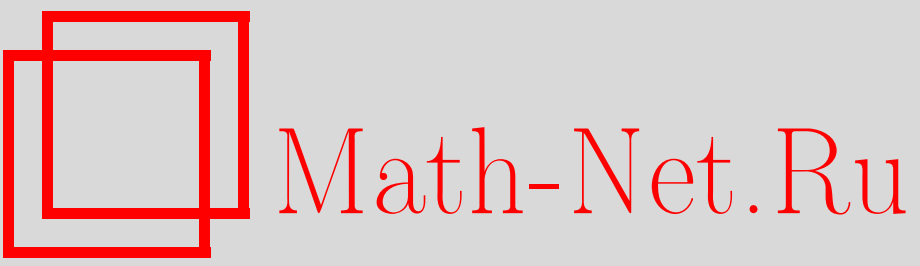

А. В. Костин, О геликоидах Дини в пространстве Минковского, Итоги науки и техн. Сер. Соврем. мат. и ее прил. Темат. обз., 2020, том 180, 50-57

DOI: https://doi.org/10.36535/0233-6723-2020-180-50-57

Использование Общероссийского математического портала Math-Net.Ru подразумевает, что вы прочитали и согласны с пользовательским соглашением

http://www.mathnet.ru/rus/agreement

Параметры загрузки:

IP: 54.84 .234 .179

26 апреля 2023 г., 15:52:57

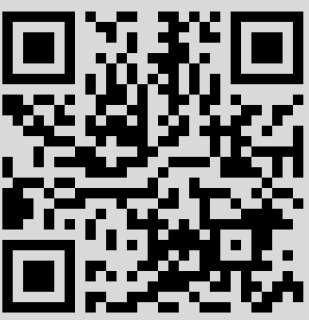


ИТОГИ НАУКИ И ТЕХНИКИ.

Современная математика и ее приложения.

Тематические обзоры.

Том 180 (2020). C. $50-57$

DOI: $10.36535 / 0233-6723-2020-180-50-57$

УДК $514.13,514.752$

\title{
О ГЕЛИКОИДАХ ДИНИ В ПРОСТРАНСТВЕ МИНКОВСКОГО
}

\author{
(c) 2020 г. $\quad$ А. В. КОСТИН
}

\begin{abstract}
АннотАция. Геликоид Дини - поверхность, полученная винтовым движением трактрисы. В paботе рассматриваются различные аналоги геликоида Дини в трехмерном пространстве Минковского. В качестве профилей берутся нетривиальные псевдоевклидовы аналоги трактрисы, отличные от псевдоевклидовых окружностей. Показано, что на аналогах геликоида Дини в псевдоевклидовом пространстве индуцируется либо метрика плоскости Лобачевского, либо метрика плоскости де Ситтера, либо вырожденная метрика.
\end{abstract}

Ключевые слова: плоскость Лобачевского, плоскость де Ситтера, геликоид Дини.

\section{ON DINI HELICOIDS IN THE MINKOWSKI SPACE}

\section{(c) 2020 A. V. KOSTIN}

\begin{abstract}
The Dini helicoid is a surface obtained by screw motion of the tractrix. In this paper, we consider various analogs of the Dini helicoid in the three-dimensional Minkowski space. As profiles, we take nontrivial pseudo-Euclidean analogs of the tractrix different from pseudo-Euclidean circles. We prove that on analogs of the Dini helicoid in a the pseudo-Euclidean space, one of the following metrics is induced: the metric of the Lobachevsky plane, the metric of the de Sitter plane, or the degenerate metric.
\end{abstract}

Keywords and phrases: Lobachevsky plane, de Sitter plane, Dini helicoid.

AMS Subject Classification: 53A35, 53B30

1. Предварительные сведения. Напомним, что трактрисой называется линия, обладающая постоянным отрезком касательной до некоторой фиксированной прямой. Геликоидом Дини (см. [7]) называется поверхность, полученная винтовым движением трактрисы (см. рис. 1):

$$
\left\{\begin{array}{l}
x=a \sin u \cos v \\
y=a \sin u \sin v \\
z=a\left(\cos u+\ln \left(\operatorname{tg} \frac{u}{2}\right)\right)+b \cdot v .
\end{array}\right.
$$

Если меридиан поверхности вращения постоянной кривизны пространства Минковского выражается с помощью элементарных функций, то он обладает постоянным отрезком касательной. Это свойство меридиана аналогично свойству меридиана поверхностей вращения постоянной кривизны в евклидовом пространстве. Интегрируемый в элементарных функциях меридиан поверхности вращения постоянной отрицательной кривизны евклидова пространства является трактрисой, постоянной положительной кривизны-окружностью. Трактриса обладает постоянным вещественным отрезком касательной до базы, окружность - постоянным мнимым отрезком касательной до изотропной прямой, проходящей через центр окружности. В пространстве 


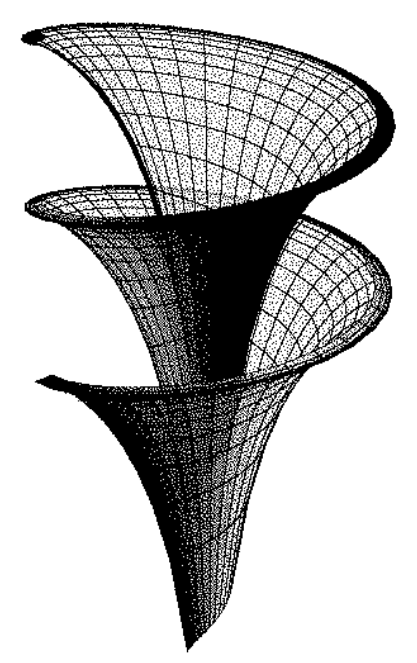

Рис. 1. Геликоид Дини

Минковского кроме окружностей с точностью до антидвижения есть еще два типа трактрис. Вид их представлен на рис. 2 и 3. Модуль отрезка касательной до оси абсцисс в стандартной псевдоевклидовой метрике $d s^{2}=\left(d x_{1}\right)^{2}-\left(d x_{2}\right)^{2}$ на обоих рисунках равен единице.

Если параметр такой трактрисы равен $a$, то ее можно задать следующими параметрическими уравнениями:

$$
\left\{\begin{array}{l}
x^{1}=a(\operatorname{sh} t-\operatorname{arctg}(\operatorname{sh} t)), \\
x^{2}=a \operatorname{ch} t .
\end{array}\right.
$$

Каждая ветвь этой трактрисы имеет изотропную асимптоту.

Другой вид трактрис может быть параметрически задан следующим образом:

$$
\left\{\begin{array}{l}
x^{1}=a\left(\ln \left(\operatorname{th} \frac{t}{2}\right)+\operatorname{ch} t\right), \\
x^{2}=a \operatorname{sh} t .
\end{array}\right.
$$

Длина отрезка касательной до оси вращения в метрике $d s^{2}=-\left(d x^{1}\right)^{2}+\left(d x^{2}\right)^{2}$ у первой кривой равна $a$, у второй - $-a \cdot i$. При вращении их также образуются поверхности постоянной кривизны с метрикой

$$
d s^{2}=a^{2} \frac{\left(d u^{1}\right)^{2} \pm\left(d u^{2}\right)^{2}}{\left(u^{2}\right)^{2}} .
$$

Касательная прямая и ось вращения для первой трактрисы являются прямыми разных типов, для второй трактрисы - прямыми одного типа.

Гауссову кривизну поверхности с невырожденной метрикой в трехмерном пространстве Минковского будем определять по формуле $K=\varepsilon h / g$, где $g, h$-дискриминанты первой и второй квадратичных форм соответственно, а $\varepsilon=\boldsymbol{n}^{2}=-\operatorname{sign} g$. Дифференциальная геометрия кривых и поверхностей трехмерного пространства Минковского изложена в работе [11]. Поверхностям вращения в этом пространстве посвящена работа [5]. Поверхности вращения эволют рассматриваемых в данной работе трактрис (см. [3]) имеют нулевую среднюю кривизну, как и соответствующая поверхность в евклидовом пространстве. Поверхности такого типа в псевдоевклидовом пространстве рассматриваются в работах $[4,12]$.

2. Геликоиды Дини в пространстве Минковского. Винтовыми движениями меридианов поверхностей вращения постоянной кривизны пространства Минковского можно получить различные аналоги геликоида Дини. Разберем несколько примеров. Всюду в данной работе будем для определенности предполагать, что $a>0, b \geqslant 0$. 


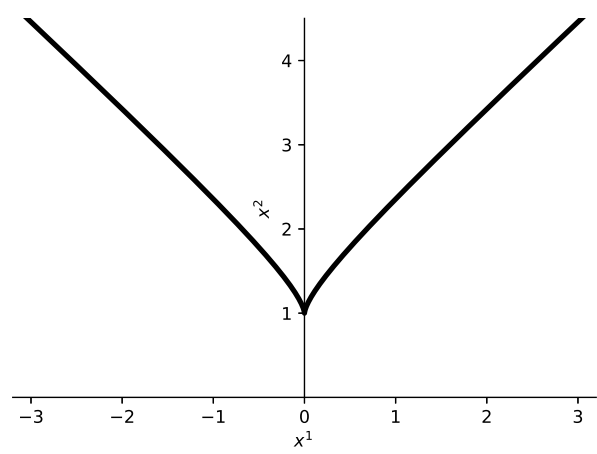

Рис. 2. Псевдоевклидова трактриса (2), у которой база и касательная являются прямыми разных типов

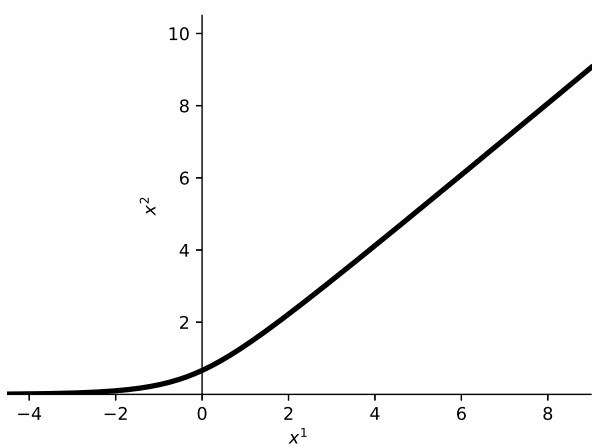

Рис. 3. Псевдоевклидова трактриса (3), у которой база и касательная являются прямыми одного типа

Вначале коснемся геликоида, полученного эллиптическим винтовым движением трактрисы (2).

Теорема 1. В трехмерном пространстве Минковского с метрикой $d s^{2}=d x^{2}+d y^{2}-d z^{2}$ рассмотрим поверхность

$$
\left\{\begin{array}{l}
x=a \operatorname{ch} u \cos v, \\
y=a \operatorname{ch} u \sin v \\
z=a(\operatorname{sh} u-\operatorname{arctg}(\operatorname{sh} u))+b \cdot v
\end{array}\right.
$$

(см. рис. 4). На поверхности (4) реализуется один из трех следующих вариантов:

(1) при $a>b$ локально реализуется геометрия Лобачевского;

(2) при $a<b$ локально реализуется геометрия плоскости де Ситтера;

(3) при $a=b$ реализуется геометрия с вырожденной метрикой.

Доказательство. Поверхность (4) получена эллиптическим винтовым движением с осью $O z$ трактрисы, у которой касательная имеет вещественную длину, а база - мнимую. Первая квадратичная форма этой поверхности имеет следующий вид:

$$
\mathrm{I}=a^{2} \operatorname{th}^{2} u d u^{2}-2 a b \operatorname{th} u \operatorname{sh} u d u d v+\left(a^{2} \operatorname{ch}^{2} u-b^{2}\right) d v^{2} .
$$

Дискриминант этой формы равен $a^{2} \operatorname{sh}^{2} u\left(a^{2}-b^{2}\right)$. При $a>b$ метрика поверхности будет положительно определенной. Рассмотрим сначала этот случай. Орт нормали поверхности при $a>b$ будет иметь следующие координаты:

$$
\boldsymbol{n}=\left(\frac{a \operatorname{sh} u \cos v-b \sin v}{\sqrt{a^{2}-b^{2}}}, \frac{a \operatorname{sh} u \sin v+b \cos v}{\sqrt{a^{2}-b^{2}}}, \frac{a \operatorname{ch} u}{\sqrt{a^{2}-b^{2}}}\right) .
$$

Вторая квадратичная форма поверхности имеет вид

$$
\mathrm{II}=-\frac{a^{2} \operatorname{th} u}{\sqrt{a^{2}-b^{2}}} d u^{2}+\frac{2 a b \operatorname{sh} u}{\sqrt{a^{2}-b^{2}}} d u d v-\frac{a^{2} \operatorname{sh} u \operatorname{ch} v}{\sqrt{a^{2}-b^{2}}} d v^{2} .
$$

Гауссова кривизна дефинитной метрики поверхности трехмерного пространства Минковского удовлетворяет условию $K=-h / g$, где $g, h$-дискриминанты первой и второй квадратичных форм соответственно. Кривизна поверхности в данном случае постоянна и равна $-1 /\left(a^{2}-b^{2}\right)$.

Подсчитаем геодезическую кривизну винтовых линий на этой поверхности. Имеем:

$$
k_{g}=\frac{a \operatorname{ch} u}{\sqrt{a^{2}-b^{2}} \sqrt{a^{2} \operatorname{ch}^{2} u-b^{2}}} .
$$

Геодезическая кривизна при всех значениях параметра $u$ больше $1 / R$, где $R=\sqrt{a^{2}-b^{2}}$, поэтому винтовые орбиты во внутренней геометрии поверхности являются окружностями. Ребру поверхности соответствует значение $u=0$. Геодезическая кривизна ребра равна $a /\left(a^{2}-b^{2}\right)$. 


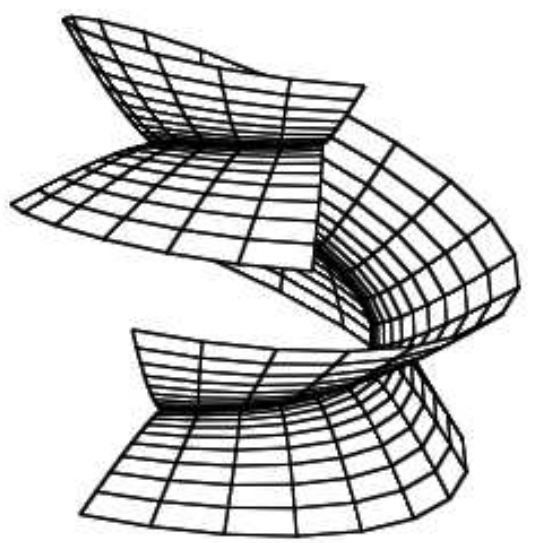

Рис. 4. Геликоид, полученный эллиптическим винтовым движением трактрисы (2)

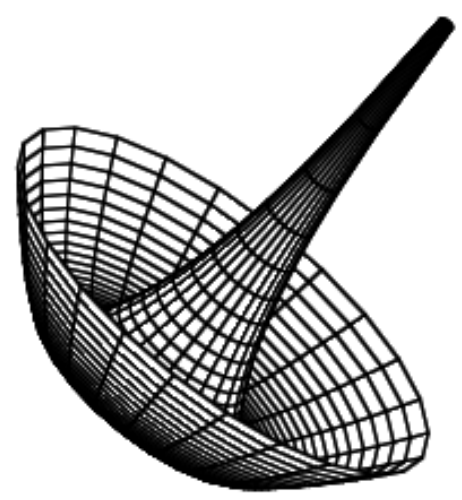

Рис. 5. «Полная» псевдосфера

Отсюда следует, что поверхность (4) изометрично накрывает плоскость Лобачевского кривизны $K=-\frac{1}{a^{2}-b^{2}}$, из которой вырезан круг радиуса $r=\sqrt{a^{2}-b^{2}} \operatorname{arcth} \frac{a}{\sqrt{a^{2}-b^{2}}}$. При $b \neq 0$ трактрисы, как и при $b=0$, являются линиями кривизны на поверхности, но уже не играют роль прямых во внутренней геометрии поверхности, и, вообще, перестают быть линиями постоянной кривизны. Ортогональные траектории трактрис, как и в евклидовом случае, являются сферическими кривыми - в данном случае кривыми на сфере вещественного радиуса трехмерного пространства Минковского - и образуют второе семейство линий кривизны на поверхности.

При $a<b$ на поверхности (4) индуцируется индефинитная метрика. Орт нормали будет иметь следующий вид:

$$
\boldsymbol{n}=\left(\frac{a \operatorname{sh} u \cos v-b \sin v}{\sqrt{b^{2}-a^{2}}}, \frac{a \operatorname{sh} u \sin v+b \cos v}{\sqrt{b^{2}-a^{2}}}, \frac{a \operatorname{ch} u}{\sqrt{b^{2}-a^{2}}}\right) .
$$

Квадрат орта нормали поверхности в этом случае равен единице. Гауссова кривизна поверхности будет определяться по формуле $K=h / g$. Формально ее значение будет иметь тот же вид: $K=-1 /\left(a^{2}-b^{2}\right)$, но кривизна метрики будет положительной. То есть, при $a<b$ на поверхности (4) индуцируется метрика идеальной области плоскости Лобачевского, или метрика де Ситтера. При $a=b$ на поверхности индуцируется вырожденная метрика. Таким образом, в зависимости от скорости трансляции при эллиптическом винтовом движении этой трактрисы мы получим три различные геометрии. Доказательство теоремы завершено.

Следствие 1. Как видно из доказательства теоремы, геометрии геликоида Дини (1) и поверхности (4) согласуются (т.е. одна поверхность является продолжением другой) только при $b=0$ (см. [1]). Случай $b=0$ изображен ниже на рис. 5. В этом случае поверхность, составленная из одной полости псевдосферы Бельтрами-Миндинга и ее псевдоевклидова продолжения, будет глобально изометрична поверхности вращения прямой вокруг параллельной ей прямой в трехмерном гиперболическом пространстве (см. [2]).

Рассмотрим теперь геликоид, полученный гиперболическим винтовым движением трактрисы (2). Оказывается, что вне зависимости от значения параметра $b$ на нем реализуется локально геометрия постоянной кривизны с индефинитной метрикой. 
Теорема 2. В трехмерном пространстве Минковского с метрикой $d s^{2}=d x^{2}+d y^{2}-d z^{2}$ рассмотрим поверхность

$$
\left\{\begin{array}{l}
x=a(\operatorname{sh} u-\operatorname{arctg}(\operatorname{sh} u)+b \cdot v), \\
y=a \operatorname{ch} u \operatorname{sh} v \\
z=a \operatorname{ch} u \operatorname{ch} v
\end{array}\right.
$$

На поверхности (5) локально реализуется геометрия плоскости де Ситтера (геометрия постоянной положительной кривизны с индефинитной метрикой). При $b \neq 0$ винтовые линии во внутренней геометрии представляют собой эквидистанты эллиптической прямой плоскости де Ситтера. При $b=0$ (чистое гиперболическое вращение) орбиты точек во внутренней геометрии представляют собой орицикль.

Доказательство. Отметим, что поверхность из формулировки теоремы получена гиперболическим винтовым движением, т.е. гиперболическим вращением с осью $O x$ и переносом вдоль этой оси трактрисы (2).

При $b=0$ имеем чистое вращение. В этом случае на поверхности индуцируется индефинитная метрика постоянной положительной кривизны $1 / a^{2}$. Покажем, что и в общем случае ситуация будет аналогичной, отличающейся лишь величиной кривизны.

Первая квадратичная форма поверхности (5) имеет вид:

$$
\mathrm{I}=-a^{2} \operatorname{th}^{2} u d u^{2}+2 a b \operatorname{th} u \operatorname{sh} u d u d v+\left(a^{2} \operatorname{ch}^{2} u+b^{2}\right) d v^{2} .
$$

Поверхность (5) допускает однопараметрическую группу изометрий и может быть изометрично отображена на поверхность вращения, параллелями которой будут орбиты однопараметрической группы. Орт нормали поверхности имеет следующие координаты:

$$
\boldsymbol{n}=\left(\frac{a \operatorname{ch} u}{\sqrt{a^{2}+b^{2}}}, \frac{a \operatorname{sh} u \operatorname{sh} v-b \operatorname{ch} v}{\sqrt{a^{2}+b^{2}}}, \frac{a \operatorname{sh} u \operatorname{ch} v-b \operatorname{sh} v}{\sqrt{a^{2}+b^{2}}}\right) .
$$

Найдя вторую квадратичную форму поверхности, убедимся, что кривизна поверхности постоянна. Действительно,

$$
\mathrm{II}=\frac{a^{2} \operatorname{th} u}{\sqrt{a^{2}+b^{2}}} d u^{2}-\frac{2 a b \operatorname{sh} u}{\sqrt{a^{2}+b^{2}}} d u d v-\frac{a^{2} \operatorname{sh} u \operatorname{ch} v}{\sqrt{a^{2}+b^{2}}} d v^{2} .
$$

Отсюда, учитывая выражение гауссовой кривизны поверхности с индефинитной метрикой через дискриминанты первой и второй квадратичных форм, получим:

$$
K=\frac{h}{g}=\frac{1}{a^{2}+b^{2}}
$$

Таким образом, на винтовой поверхности (5) также индуцируется метрика плоскости де Ситтера, или метрика идеальной области плоскости Лобачевского. Зависимость между кривизной и скоростью трансляции вдоль оси $O z$ такая же, как у геликоида Дини в евклидовом пространстве. Кривизна его равна $-1 /\left(a^{2}+b^{2}\right)$. Ребро геликоида Дини при $b \neq 0$ во внутренней геометрии поверхности является эквидистантой, как, впрочем, и все остальные винтовые линии, являющиеся орбитами точек евклидовой трактрисы. Параметр эквидистанты при удалении от ребра уменьшается. В соответствии с этим при удалении от ребра (при стремлении $u$ к бесконечности) геодезическая кривизна орбиты стремится к нулю. Под геодезической кривизной кривой в данной работе всюду понимается модуль проекции вектора кривизны на касательную плоскость.

Вернемся к поверхности (5). Найдем геодезическую кривизну винтовых линий на этой поверхности. Имеем:

$$
k_{g}=\frac{a \operatorname{ch} u}{\sqrt{a^{2}+b^{2}} \sqrt{a^{2} \operatorname{ch}^{2} u+b^{2}}} .
$$

Ребру поверхности соответствует значение $u=0$. Для этого значения $k_{g}=a /\left(a^{2}+b^{2}\right)<1 / R$, где $R=\sqrt{a^{2}+b^{2}}$. То есть, как и для геликоида Дини, при ненулевой скорости трансляции граничный орицикл превращается в эквидистанту. В данном случае это будет эквидистанта эллиптической прямой плоскости де Ситтера. Кривизна эквидистанты эллиптической прямой, удаленной от нее 
на расстояние $h \cdot i$, будет равна $\frac{1}{R}$ th $\frac{h}{R}$, если кривизна плоскости де Ситтера равна $1 / R^{2}$. Отсюда следует, что в индуцированной на винтовой поверхности (5) геометрии плоскости де Ситтера граничное ребро удалено от эллиптической прямой на расстояние $i \sqrt{a^{2}+b^{2}}$ arth $\frac{a}{\sqrt{a^{2}+b^{2}}}$. Следует отметить, что самой эллиптической прямой среди винтовых орбит нет. Данная винтовая поверхность вообще не содержит ни одной полной прямой эллиптического, гиперболического или изотропного типа.

Замечание 1. Теорема Бура (см. [6]) гласит, что любая винтовая поверхность в евклидовом пространстве изометрична поверхности вращения. Аналогам этой теоремы в псевдоевклидовом пространстве посвящены работы (см. [8-10]). Для каждой из рассматриваемых в данной работе винтовых поверхностей можно построить изометричную поверхность вращения. Покажем это на примере поверхности (5).

Доказательство. Зададим в плоскости $O x z$ меридиан:

$$
\left\{\begin{array}{l}
x=\int \sqrt{\frac{R^{2}\left(1+B^{2}\right)-z^{2}}{R^{2} B^{2}-z^{2}}} d z, \quad z^{2} \geqslant R^{2}\left(1+B^{2}\right), \\
y=0
\end{array}\right.
$$

Рассмотрим гиперболическое вращение этой кривой вокруг оси $O x$. После введения псевдоевклидовых цилиндрических координат

$$
x=x(\rho), \quad y=\rho \operatorname{sh} \varphi, \quad z=\rho \operatorname{ch} \varphi .
$$

Индуцированная на поверхности вращения метрика примет вид

$$
d s^{2}=\left(\left(x_{\rho}^{\prime}\right)^{2}-1\right) d \rho^{2}+\rho^{2} d \varphi^{2} .
$$

В данном случае получим:

$$
d s^{2}=\frac{R^{2}}{R^{2} B^{2}-\rho^{2}} d \rho^{2}+\rho^{2} d \varphi^{2} .
$$

Введем на поверхности новые координаты:

$$
\rho=R B \operatorname{ch} \frac{r}{R}, \quad \varphi=\frac{\omega}{R B} .
$$

Тогда метрика на поверхности примет вид

$$
d s^{2}=-d r^{2}+\operatorname{ch}^{2} \frac{r}{R} d \omega^{2} .
$$

Подставив в эту формулу значение $R=\sqrt{a^{2}+b^{2}}$, получим:

$$
d s^{2}=-d r^{2}+\operatorname{ch}^{2} \frac{r}{\sqrt{a^{2}+b^{2}}} d \omega^{2} .
$$

Меридианы поверхности имеют мнимую длину, параллели - вещественную. Параллели представляют собой семейство эквидистант эллиптической прямой, меридианы - пучок гиперболических прямых, ортогональных этой эллиптической прямой и всем ее эквидистантам. Именно на такую поверхность глобально изометрично отображается поверхность (5) при $b \neq 0$.

Перейдем к рассмотрению винтовых движений трактрисы (3).

Теорема 3. Рассмотрим геликоид, полученный эллиптическим винтовым движением трактрисы (3) в пространстве Минковского (см. рис. 7):

$$
\left\{\begin{array}{l}
x=a \operatorname{sh} u \cos v \\
y=a \operatorname{sh} u \sin v \\
z=a\left(\ln \left(\operatorname{th} \frac{u}{2}\right)+\operatorname{ch} u\right)+b \cdot v .
\end{array}\right.
$$

При всех значениях параметра b данная поверхность несет на себе локальную геометрию плоскости де Cuтmepa. 


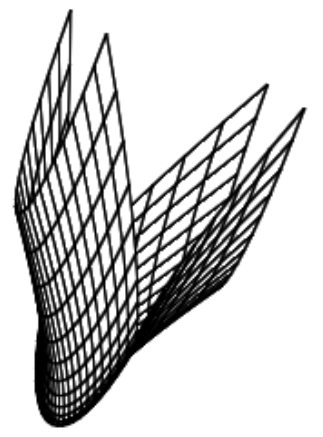

Рис. 6. Геликоид, полученный винтовым гиперболическим движением трактрисы (2)

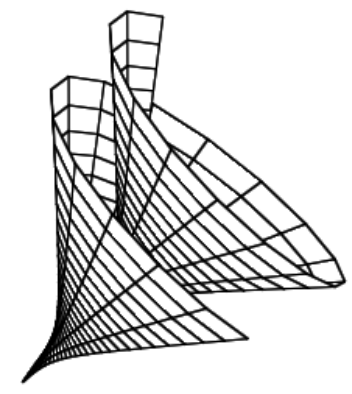

Рис. 7. Геликоид, полученный винтовым эллиптическим движением трактрисы (3)

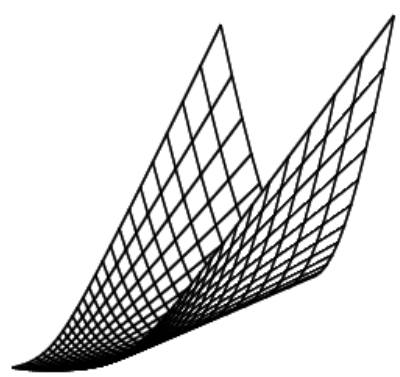

Рис. 8. Геликоид, полученный винтовым гиперболическим движением трактрисы (3)

Доказательство. Первая квадратичная форма поверхности имеет вид:

$$
\mathrm{I}=-a^{2} \operatorname{cth}^{2} u d u^{2}-2 a b \operatorname{cth} u \operatorname{ch} u d u d v+\left(a^{2} \operatorname{sh}^{2} u-b^{2}\right) d v^{2} .
$$

Дискриминант этой формы равен $-a^{2} \mathrm{ch}^{2} u\left(a^{2}+b^{2}\right)$. Метрика поверхности индефинитна. Для вычисления кривизны найдем вторую квадратичную форму. Орт псевдоевклидовой нормали имеет такие координаты:

$$
\boldsymbol{n}=\left(\frac{a \operatorname{ch} u \cos v-b \sin v}{\sqrt{a^{2}+b^{2}}}, \frac{a \operatorname{ch} u \sin v+b \cos v}{\sqrt{a^{2}+b^{2}}}, \frac{a \operatorname{sh} u}{\sqrt{a^{2}+b^{2}}}\right) .
$$

Вторая квадратичная форма поверхности имеет вид

$$
\mathrm{II}=\frac{a^{2} \operatorname{cth}^{2} u}{a^{2}+b^{2}} d u^{2}+\frac{2 a b \operatorname{ch} u}{\sqrt{a^{2}+b^{2}}} d u d v-\frac{a^{2} \operatorname{sh} u \operatorname{ch} v}{\sqrt{a^{2}+b^{2}}} d v^{2} .
$$

Отсюда следует, что кривизна поверхности постоянна и равна $1 /\left(a^{2}+b^{2}\right)$.

В заключение рассмотрим гиперболическое винтовое движение трактрисы (3). Оказывается, что эта ситуация близка к ситуации, описанной в теореме 1.

Теорема 4. Зададим в пространстве Минковского следующую поверхность-геликоид, полученный гиперболическим винтовым движением трактрисы (3) (см. рис. 8):

$$
\left\{\begin{array}{l}
x=a \ln \left(\operatorname{th} \frac{u}{2}\right)+\operatorname{ch} u+b \cdot v \\
y=a \operatorname{sh} u \operatorname{sh} v \\
z=a \operatorname{sh} u \operatorname{ch} v
\end{array}\right.
$$

Возможен один из следующих трех случаев:

(1) при $a>b$ реализуется геометрия плоскости Лобачевского;

(2) при $a<b$ реализуется геометрия плоскости де Ситтера;

(3) при $a=b$ реализуется геометрия с вырожденной метрикой.

Доказательство. Если $b=0$, то получим чистое гиперболическое вращение трактрисы (3). На поверхности в этом случае индуцируется метрика плоскости Лобачевского. Меридианы поверхности образуют пучок параллельных прямых этой плоскости, параллели - пучок ортогональных им орициклов. Поверхность глобально изометрична плоскости Лобачевского. Рассмотрим случай общего винтового движения. Первая квадратичная форма поверхности имеет такой вид:

$$
\mathrm{I}=a^{2} \operatorname{cth}^{2} u d u^{2}+2 a b \operatorname{cth} u \operatorname{ch} u d u d v+\left(a^{2} \operatorname{sh}^{2} u+b^{2}\right) d v^{2} .
$$


Дискриминант этой формы равен $a^{2} \mathrm{ch}^{2} u\left(a^{2}-b^{2}\right)$. При $a>b$ метрика на поверхности будет положительно определенной. Орт псевдоевклидовой нормали будет иметь такие координаты:

$$
\boldsymbol{n}=\left(\frac{a \operatorname{sh} u}{\sqrt{a^{2}-b^{2}}}, \frac{a \operatorname{ch} u \sin v-b \operatorname{ch} v}{\sqrt{a^{2}-b^{2}}}, \frac{a \operatorname{ch} u \operatorname{ch} v-b \operatorname{sh} v}{\sqrt{a^{2}-b^{2}}}\right) .
$$

Вторая квадратичная форма поверхности имеет вид:

$$
\mathrm{II}=-\frac{a^{2} \operatorname{cth}^{2} u}{\sqrt{a^{2}-b^{2}}} d u^{2}-\frac{2 a b \operatorname{ch} u}{\sqrt{a^{2}-b^{2}}} d u d v-\frac{a^{2} \operatorname{sh} u \operatorname{ch} v}{\sqrt{a^{2}-b^{2}}} d v^{2} .
$$

Отсюда следует, что в данном случае кривизна поверхности также постоянна и равна $-1 /\left(a^{2}-b^{2}\right)$.

Подсчитаем геодезическую кривизну винтовых линий на этой поверхности. Имеем:

$$
k_{g}=\frac{a \operatorname{sh} u}{\sqrt{a^{2}-b^{2}} \sqrt{a^{2} \operatorname{sh}^{2} u+b^{2}}} .
$$

Геодезическая кривизна меньше, чем $1 / \sqrt{a^{2}-b^{2}}$, поэтому винтовые линии во внутренней геометрии являются эквидистантами. Одно семейство линий кривизны образуют трактрисы, второе семейство - ортогональные им сферические кривые. Трактрисы при $b \neq 0$ перестают быть геодезическими во внутренней геометрии поверхности.

При $a=b$ метрика на поверхности становится вырожденной, при $a<b$ метрика становится индефинитной. Таким образом, в последнем случае на поверхности опять индуцируется метрика де Ситтера, поскольку кривизна поверхности остается постоянной.

\section{СПИСОК ЛИТЕРАТУРЫ}

1. Костин А. В. Геликоиды Дини в пространстве Минковского// Междунар. конф. «Дни геометрии в Новосибирске-2013», 2013. - С. 52.

2. Костин А. В. Об асимптотических линиях на псевдосферических поверхностях// Владикавказ. мат. ж. -2019 . -21 , № 1. - С. 14-24.

3. Костин А. В., Костина Н. Н. Об эволютах некоторых кривых на псевдоевклидовой плоскости// в кн.: Тр. Междунар. школы-семинара по геометрии и анализу памяти Н. В. Ефимова, 2004. - С. $34-$ 35 .

4. Albujer A. L., Caballero M. Geometric properties of surfaces with the same mean curvature in $\mathbb{R}^{3}$ and $\mathbb{L}^{3} / /$ J. Math. Anal. Appl. — 2017. - 445, № 1. - P. 1013-1024.

5. Barros M., Caballero M., Ortega M. Rotational surfaces in $\mathbb{L}^{3}$ and solutions of the nonlinear sigma model// Commun. Math. Phys. - 2009. - 290, № 2. - P. 437-477.

6. Bour E. Memoire sur le deformation de surfaces// J. Ecole Polytechn. - 1862. - 39. - P. 1-148.

7. Dini $U$. Sur les surface à courbure constante négative et les surfaces applicables sur les surfaces à aire minima// C. R. Acad. Sci. - 1865. - P. 340-341.

8. Güler E., Vanli A. T. Bour's theorem in Minkowski 3-space// J. Math. Kyoto Univ. - 2006. — 46, № 1. - P. 47-63.

9. Ikawa T. Bour's theorem in Minkowski geometry// Tokyo J. Math. — 2001. — 24, № 2. — P. 377-394.

10. Ji F., Kim Y. H. Isometries between minimal helicoidal surfaces and rotation surfaces in Minkowski space// Appl. Math. Comput. - 2013. - 220. - P. 1-11.

11. Lopez R. Differential geometry of curves and surfaces in Lorentz-Minkowski space// Int. Electron. J. Geom. - 2014. - 7, № 1. - P. 44-107.

12. Lopez R., Kaya S. New examples of maximal surfaces in Lorentz-Minkowski space// Kyushu J. Math. 2017. - 71, № 2. - P. 311-327.

Костин Андрей Викторович

Казанский (Приволжский) федеральный университет, Елабужский институт, Елабуга

E-mail: kostin_andrei@mail.ru 